\title{
High expression of small GTPase Rab3D promotes cancer progression and metastasis
}

\author{
Jian Yang1,2,3, Wei Liu1,2,3, Xin'an Lu'1,2,3, Yan Fu'1,2,3, Lin Li ${ }^{1,2,3}$ and Yongzhang Luo ${ }^{1,2,3}$ \\ ${ }^{1}$ National Engineering Laboratory for Anti-tumor Protein Therapeutics, Tsinghua University, Beijing, China \\ 2 Beijing Key Laboratory for Protein Therapeutics, Tsinghua University, Beijing, China \\ ${ }^{3}$ Cancer Biology Laboratory, School of Life Sciences, Tsinghua University, Beijing, China \\ Correspondence to: Yongzhang Luo, email: yluo@mail.tsinghua.edu.cn \\ Keywords: Rab3D, EMT, exosomes, Hsp90a, tumor metastasis
}

Received: February 02, $2015 \quad$ Accepted: February 21, $2015 \quad$ Published: March 14, 2015

This is an open-access article distributed under the terms of the Creative Commons Attribution License, which permits unrestricted use, distribution, and reproduction in any medium, provided the original author and source are credited.

\section{ABSTRACT}

Rab GTPases control exocytic and endocytic membrane trafficking such as exosomes release. As a secretory small GTPase, Rab3D is a vital regulator for protein secretion. However, the role of Rab3D in cancer was never systematically studied. The aim of this study is to examine its function and mechanism in cancer, especially metastasis. We detected protein levels of Rab3D in nine cancer cell lines and twelve types of clinical cancer specimens. Subsequently, we established in vitro migration and in vivo orthotopic metastatic mouse models to study the role of Rab3D in tumor metastasis. Here, we reported that the expression levels of Rab3D were dysregulated in cancer cells and highly correlated with tumor malignancies in the clinical samples. Increased expressions of Rab3D led to tumor invasion in vitro and lung metastasis in vivo, whereas Rab3D knockdown suppressed the tumor cell motility. Mechanistic studies revealed that Rab3D activated intracellular the AKT/GSK3 $\beta$ signaling to induce the EMT process. In addition, it also regulated the extracellular secretion of Hsp90a to promote tumor cell migration and invasion. These results prove that Rab3D is a key molecule to regulate tumor metastasis, suggesting that blocking the Rab3D function can be a potential therapeutic approach for cancer metastasis.

\section{INTRODUCTION}

More than $90 \%$ of cancer mortality is attributable to metastases, thus the prevention of metastasis guides the development of novel diagnostic and therapeutic strategies [1]. In the tumor microenvironment, cell migration and invasion are regulated by many factors such as intracellular signaling pathways, growth factors or oncoproteins [2]. In recent years, increasing numbers of studies have revealed that vesicular exocytosis can also play an essential role in tumor progression and malignancy [3].

Exosomes, characterized by a size of $30-100 \mathrm{~nm}$ in diameter, have been viewed as secreted vesicles that enable extracellular communications [4-6] and have played an important role in tumor progression and metastasis by promoting the extracellular matrix degradation and also by remodeling to establish the metastasis niche [7, 8]. Abundant proteins, including membrane trafficking molecules, cytoskeleton molecules, chaperones, signal transduction proteins and cytoplasmic enzymes have been included in secreted exosomes [9].

Rab GTPases, as intracellular transport proteins, are master regulators of exocytic and endocytic membrane trafficking [10]. The different secretory processes are controlled by corresponding Rab proteins including Rab3A/B/C/D, Rab26, Rab27A/B and Rab37. Aberrant expressions of Rabs can influence cancer development and metastasis [11]. Kim and coworkers have shown that $\mathrm{Rab} 3 \mathrm{~A}$ is a novel oncogene involved in glioma initiation and progression via promoting cell proliferation [12]. Another Rab3 subfamily member Rab3B has been reported to be overexpressed in prostate cancer patients, which reduces tumor cell death [13]. Rab37 acts as a metastasis-related tumor suppressor gene in lung cancer, and low mRNA expression of Rab37 is significantly associated with lung metastasis [14]. Besides, Rab27 has been reported to control essential steps of the exosomes secretion pathway [15]. Rab27A promotes tumor 
progression by mediating the secretion of cytokines and exosomes in tumor microenvironment [16]. At the meantime, high level of Rab27B has been found in a poor prognostic phenotype of human breast cancer, which is due to the regulatory effect on invasive growth and metastasis [17-19]. In Rab27 regulated exosomes, the indispensable and ubiquitous molecular chaperone Hsp90 $\alpha$ has been found to enhance cancer cell invasion through activating matrix metalloproteinases (MMPs) $[17,20]$. Hsp90 $\alpha$, an isoform of the Hsp90 family, can be translocated to the cell surface [21] and secreted into the extracellular space $[22,23]$. Our previous studies have demonstrated that the level of plasma Hsp90 $\alpha$ is positively correlated with the tumor malignancy in clinical cancer patients and the secreted Hsp90 $\alpha$ stabilizes MMP-2 to facilitate tumor invasion $[24,25]$. These above reports suggest that the abnormal expression of secretory Rab GTPases could be a generalized feature of tumor progression.

Rab3D is another very important member of the secretory Rab GTPases. Comparing to Rab26 and Rab37, which modulate the secretion in specialized cell types, Rab3D is enriched in non-neuronal tissues, whereas $\mathrm{Rab} 3 \mathrm{~A} / \mathrm{B} / \mathrm{C}$ are mostly expressed in the nervous system. In addition, the $\mathrm{N}$ - and $\mathrm{C}$-terminal regions of Rab3D are largely distinct from other Rab3 isoforms. Rab3D regulates exocytosis processes and apically directs transcytosis [26-28]. Although Rab3D is widely expressed in several secretory tissues and even in rat pancreatic acinar tumor cell line AR42J cells [29], the biological function and molecular mechanisms for Rab3D in cancer have not been elucidated so far.

Here, we demonstrated that Rab3D promotes breast cancer cell invasion and lung metastasis by EMT induction through the activation of the AKT/GSK-3 $\beta /$ Snail signaling pathway. More importantly, a significant positive relationship between Rab3D and the malignancy of cancer patients is observed. Thus, this study provides a novel insight into the biomedical relevance of Rab3D in tumor malignancies, which indicates that Rab3D plays a critical role in promoting tumor metastasis and is a promising therapeutic target for the treatment of cancer.

\section{RESULTS}

\section{Rab3D is aberrantly elevated in human cancers and correlated with the malignancy}

To explore the role of Rab3D in tumor progression, we firstly analyzed the level of Rab3D in cancer cells. The levels of intracellular Rab3D in many types of cancer cells were significantly higher than that in the immortalized human microvascular endothelial cells (HMEC) (Fig. $1 \mathrm{~A}-\mathrm{C})$. In addition, in breast cancer cells with different malignancies, the levels of intracellular Rab3D were highly elevated in invasive SKBr-3 and MDA-MB-231 cell lines compared with that in the non-invasive MCF7 (Fig. 1A), showing a positive correlation between breast cancer cell aggressive phenotypes and Rab3D expression levels. Also in melanoma cell lines, the levels of intracellular Rab3D were also aberrantly up-regulated in more malignant F10 cancer cells (Fig. 1B). And in other type of cancer cells such as lung cancer, the high expression of Rab3D was also observed (Fig. 1C).

Next, we examined the Rab3D expression in clinical cancer patients' samples using immunohistochemistry on a breast cancer tissue microarray containing 50 specimens. Tissues were scored on the basis of staining intensities of Rab3D expressions and the percentages of cancer cells stained. We found that $72.2 \%$ (26/36) malignant breast cancer tissues showed the positive staining, whereas the intensity of Rab3D staining in normal tissues or benign breast tumors was negative (Fig. 1D-E, Fig. S1A and Table $\mathrm{S} 1$ ). More intriguingly, similar to breast cancer tissue, high expression levels of Rab3D were also observed in other types of cancers including prostate, lung, colon, ovary, liver, uterine cervix, esophagus and skin carcinoma tissues (Fig. S1C and Table S2), indicating that Rab3D may play an important and critical role in the progression of these tumor types.

To further confirm the clinical relevance that Rab3D is positively correlated with tumor malignancy, we detected the level of Rab3D using another breast tissue microarray containing 110 cases with known clinical records and analyzed Rab3D's association with clinical and pathological parameters including staging and grading. Notably, IHC staining showed that Rab3D was markedly increased in stage III-IV $(P=0.002)$, but not related to tumor grade $(P=0.208)$, showing a significant correlation between Rab3D expressions and cancer TNM stages (Fig. 1F-G, Fig. S1B and Table S3).

\section{Rab3D regulates cancer cell motility and invasiveness}

To investigate whether Rab3D is required for the invasive phenotypes of cancer cells, we knocked down Rab3D in highly invasive MDA-MB-231 cells and highly expressed Rab3D in non-invasive MCF-7 cells, respectively. The Western Blot analysis proved the efficiencies of siRNA-mediated Rab3D knockdown and its overexpression (Fig. S2A-B).

Cell motility driven by cytoskeletal rearrangement plays an essential role in the development of metastasis [30]. We thus explored the effect of Rab3D on actin cytoskeleton. As shown in Fig. 2A and Fig. S2C, Rab3D knockdown clearly inhibited the membrane ruffling formation and shrank the elongated morphology. Furthermore, we found that MCF-7 cells transfected with GFP-Rab3D formed the cellular extension and spreading 
A

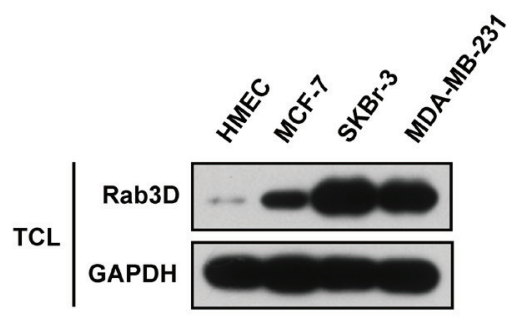

D

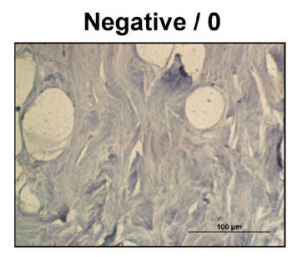

Positive / 2+

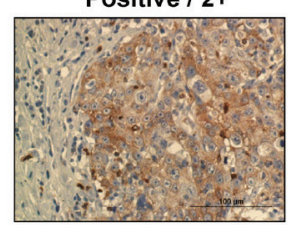

$\mathbf{F}$

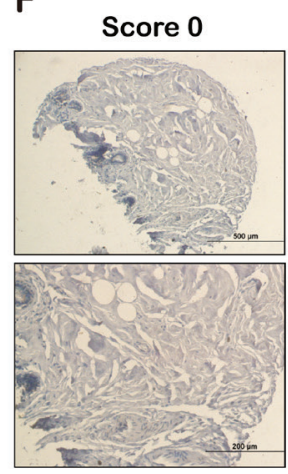

Score 2+
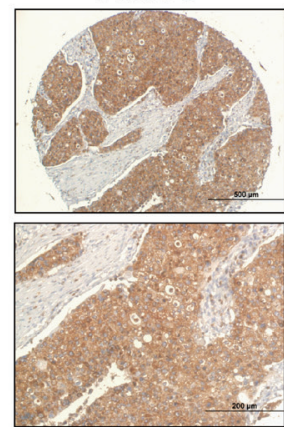

B

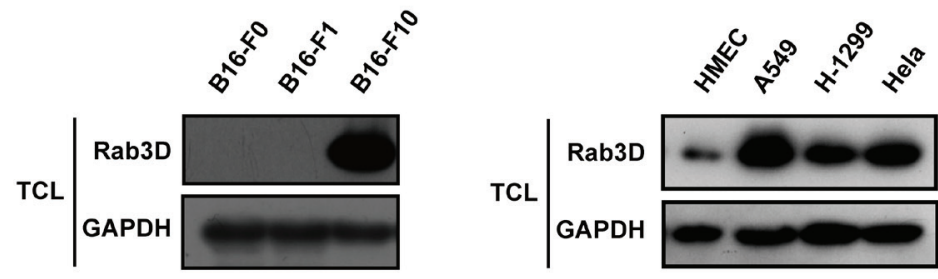

$E$

\begin{tabular}{lccccc}
\hline Variable & Score $\mathbf{0}$ & Score $\mathbf{1}$ & Score 2 & Score 3 & chi-square test \\
\hline $\begin{array}{l}\text { Total No. } \\
\text { of patients }\end{array}$ & 11 & 13 & 15 & 11 & $\mathrm{P}<0.001$ \\
Normal & 6 & 0 & 0 & 0 & \\
Benign & 0 & 8 & 0 & 0 & \\
Malignant & 5 & 5 & 15 & 11 & \\
\hline
\end{tabular}

G

\begin{tabular}{lccccc}
\hline \multicolumn{1}{c}{ Variable } & Score 0 & Score 1 & Score 2 & Score 3 & chi-square test \\
\hline $\begin{array}{l}\text { Total No. } \\
\text { of patients }\end{array}$ & 18 & 39 & 34 & 19 & $\mathrm{P}<0.001$ \\
$\begin{array}{l}\text { Normal } \\
\text { Malignant }\end{array}$ & 10 & 0 & 0 & 0 & \\
Stage & 8 & 39 & 34 & 19 & \\
$\quad$ I & 2 & 7 & 4 & 1 & \\
II & 6 & 27 & 27 & 8 & \\
III-IV & 0 & 5 & 3 & 10 & \\
Grade & & & & & $\mathrm{P}=0.208$ \\
1 & 1 & 12 & 4 & 4 & \\
2 & 6 & 25 & 22 & 13 & \\
3 & 0 & 2 & 5 & 0 & \\
\hline
\end{tabular}

Figure 1: Increased Rab3D in malignant tumor cells and in clinical cancer specimens. (A). Western Blot analysis of intracellular Rab3D expression in HMEC, non-invasive breast cancer MCF-7, and invasive SKBr-3, MDA-MB-231 cell lines. (B). Western Blot analysis of intracellular Rab3D expression in melanoma F0, F1, F10 cell lines. (C) Western Blot analysis of intracellular Rab3D expression in lung cancer A549, H-1299 cell lines and ovary cancer Hela cell line. (D). Representative Rab3D staining in normal tissues, benign tumor and malignant breast cancer samples that illustrate immunohistochemical scores of $0,1,2$ and 3. Scale bar, $100 \mu \mathrm{m}$. (E). Association of Rab3D immunohistochemical scores with tumor malignancy. The $\boldsymbol{x}^{2}$ test was used to test difference between categorical variables. (F). Representative Rab3D staining in different stages of malignant breast cancer samples that illustrate immunohistochemical scores of 0, 1, 2 and 3. Scale bar, $100 \mu \mathrm{m}$. (G). Associations of Rab3D immunohistochemical scores with tumor stage (I, II, III-IV) and tumor grade $(1,2$, and 3$)$. The $\mathrm{x}^{2}$ test was used to test difference between categorical variables. 

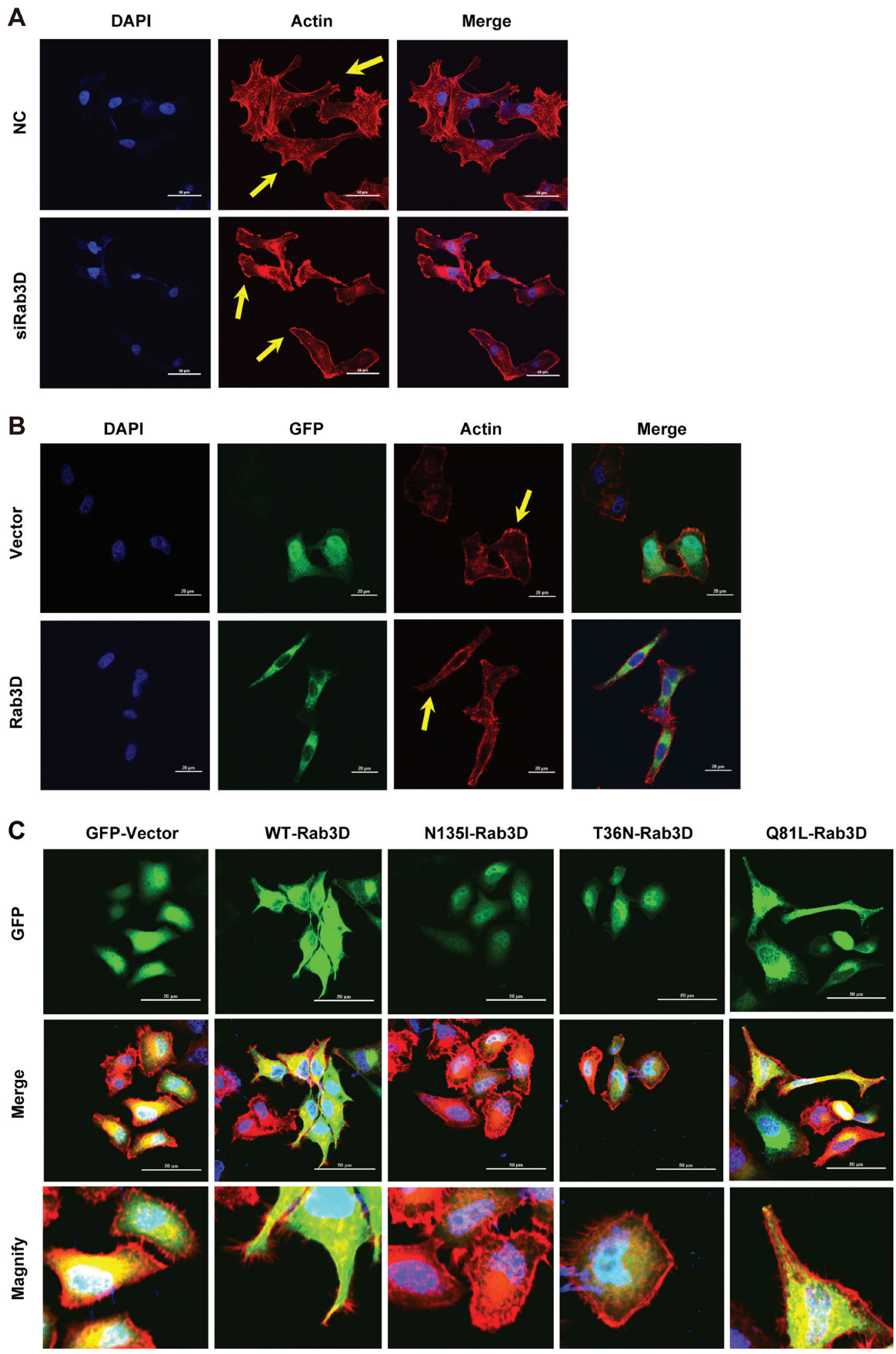

Figure 2: The effect of Rab3D on tumor cells morphology and ruffle formation. (A). The effect of Rab3D on cytoskeletal rearrangements. Control and siRab3D MDA-MB-231 cells were kept in serum and then fixed. Membrane ruffles formation was analyzed by confocal microscope using a Rhodamine labeled phalloidin antibody to visualize actin cytoskeleton. Scale bar, $50 \mu \mathrm{m}$. (B). The effect of overexpressing Rab3D on tumor cell morphology. MCF-7 cells transiently transfected with GFP or GFP-Rab3D-expressing plasmid. Nuclei were stained with DAPI and actin was stained with Rhodamine labeled phalloidin antibody. (C). The effect of different Rab3D mutants on tumor cell morphology. Rab3D (Green), Actin (Red), DAPI (Blue). Scare: $50 \mu \mathrm{m}$. 
protrusion, whereas the GFP control group exhibited no morphological change (Fig. 2B and S2D). Rab3D is a small GTP-binding protein and acts as the molecular switch between the GDP-bound inactive and GTP-bound active forms. We therefore constructed three mutations of Rab3D. N135I-Rab3D and T36N-Rab3D are both dominant negative mutants which are defective in the GTP binding. Q81L-Rab3D is a constitutively active mutant defective in the GTP hydrolysis [31]. MCF-7 cells transfected with N135I-Rab3D and T36N-Rab3D did not change their morphologies, while Q81L-Rab3Dexpressing cells showed elongated protrusions (Fig. 2C), indicating that Rab3D enhances tumor cell motility depending on its GTP binding activity.

To ascertain the role of Rab3D in tumor motility, wound healing assays were carried out. The migration abilities were markedly repressed in MDA-MB-231 cells transfected with Rab3D siRNA (Fig. 3A). Similar inhibition effects were obtained when detecting the invasion capabilities of tumor cells on Matrigelcoated transwell inserts in the invasion assay (Fig. 3B). siRab3D knockdown-reduced migration and invasion were also observed in other cell line such as melanoma A375 cells (Fig. 3A and 3B). Matrix metalloproteinases (MMPs) have played important roles in the degradation of the extracellular matrix to promote tumor invasion and metastasis [32]. MMP-9 activity was significantly decreased when knocking down Rab3D (Fig. 3C). In contrast, the overexpression of Rab3D or Q81L-Rab3D in MCF-7 enhanced cell motility and invasiveness dramatically (Fig. 3D-E, Fig. S2E-G). Taken together, these results prove that Rab3D is indispensable for cell migration and invasion in vitro in a GTP dependent manner.

\section{Rab3D induces epithelial to mesenchymal transition}

To unravel the molecular mechanisms by which Rab3D regulates cancer progression, we focused on the epithelial to mesenchymal transition (EMT) induction, because the process of EMT has been documented to be associated with tumor progression and metastasis [33].

It has been reported that the level of mesenchymal marker N-cadherin in MDA-MB-231 cells is highly elevated compared with MCF-7 cells [34]. To uncover whether Rab3D induces EMT process, we detected EMT marker in Rab3D-MCF-7 cells and siRab3DMDA-MB-231 cells, respectively. As expected, Rab3D overexpression increased $\mathrm{N}$-cadherin expression while decreased the level of epithelial marker E-cadherin (Fig. 4A). Conversely, Rab3D knockdown resulted in a decreased $\mathrm{N}$-cadherin expression level, but increased the level of E-cadherin in siRab3D-MDA-MB-231 cells (Fig. 4B). Furthermore, the expression levels of phosphorylated
AKT, phosphorylated GSK3 $\beta$, ZEB-1 and snail-1, all of which are very important for EMT process during cancer progression, were significantly up regulated in Rab3DMCF-7 cells, but decreased in siRab3D-MDA-MB-231 cells (Fig. 4C).

\section{Rab3D regulates $\mathrm{Hsp} 90 \alpha$ secretion to promote tumor cells invasion}

To further investigate the mechanism how Rab3D promotes cells invasion, we firstly detected the effect of Rab3D on the release of exosomes. Extracellular exosomes were isolated by sequential centrifugation (Fig. 5A). The size range of exosomes and their average diameter were not changed after overexpressing Rab3D (Fig. 5B), whereas the number of released exosomes was significantly increased (Fig. 5C). In addition, Rab3D overexpression resulted in the elevated concentration of the exosomes protein extracts and the level of exosomal markers such as Alix and CD63, conversely, siRNAmediated knockdown of Rab3D reversed this phenotype (Fig. 5D-E). These data suggest that Rab3D controls the release of exosomes.

Secreted Hsp90 $\alpha$ plays an essential role in tumor invasion [24, 35]. Our group has shown that the level of plasma Hsp90 $\alpha$ is positively correlated with tumor malignancy in patients samples [24]. Clinical trials with the enrollment of 2,347 cases validated plasma Hsp90 $\alpha$ as a novel tumor biomarker. The ELISA kit for Hsp90 $\alpha$ detection has been approved by China Food and Drug Administration (CFDA) and obtained the European Union $\mathrm{CE}$ and ISO13485 certifications, respectively. It has been reported that Hsp90 $\alpha$ exists in exosomes and its secretion can be modulated by Rab27 [17, 20], we thus wondered whether Rab3D also regulate Hsp90 $\alpha$ secretion.

Rab3D can dramatically facilitate the secretion of Hsp90 $\alpha$ compared with other Rabs such as Rab11A, Rab11B, Rab27A, Rab27B, Rab35 and Rab37 (Fig. S3H and S3I). Moreover, the knockdown of Rab3D in MDAMB-231 cells could reduce Hsp90 $\alpha$ secretion compared with the control cells (Fig. S3A). Western Blot and Mass Spectrometry analysis confirmed that Hsp90 $\alpha$ was among the up-regulated proteins in the CM of Rab3D-MCF-7 cells (Fig. S3B and S3C). Besides, the secretion of Hsp90 $\alpha$ is dependent on the GTPase function of Rab3D (Fig. 5F). These results demonstrated that Rab3D indeed regulated Hsp90 $\alpha$ secretion.

Secreted Hsp90 $\alpha$ is known to be essential in cancer cell invasion through stabilizing extracellular proteins such as MMPs or active intracellular signaling [36]. We found that the loss of migration and invasion by neutralization of secreted Hsp90 $\alpha$ with its antibodies cannot be rescued by overexpression of Rab3D (Fig. 5G-5H and Fig. S3D-E). In accordance, the abrogation of the decreased migration and invasion in the cells treated with siRNA of Rab3D 
A
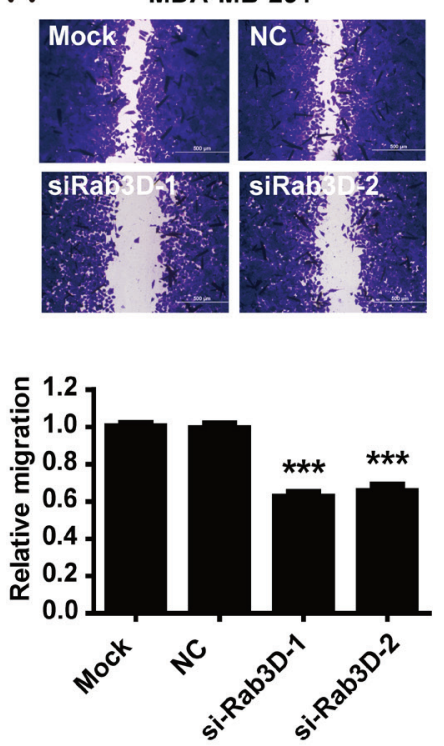

C

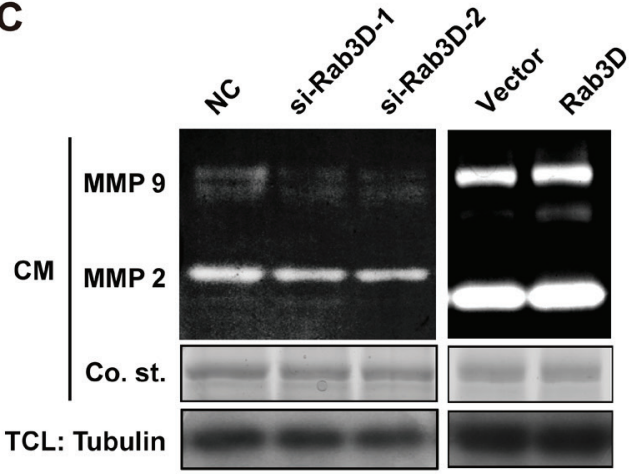

E

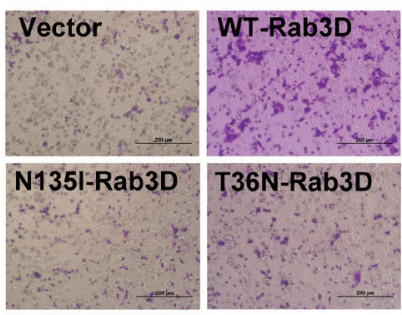

Q81LRáb3D

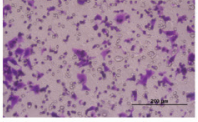

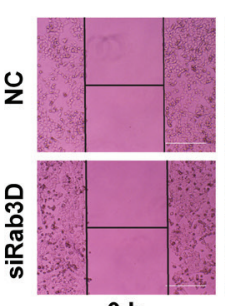

$\mathbf{O} \mathbf{h}$
A375

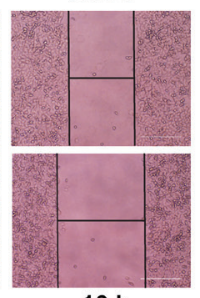

$16 \mathrm{~h}$
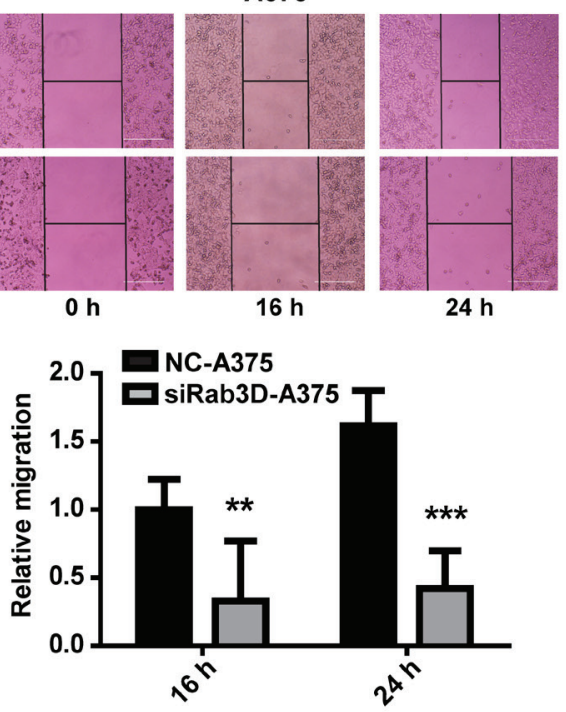

B MDA-MB-231

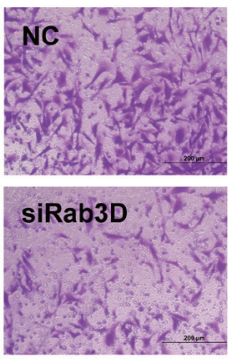

A375

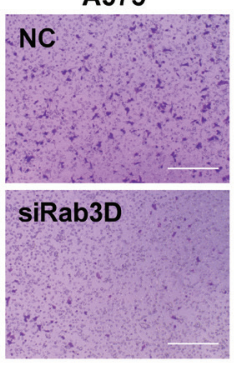

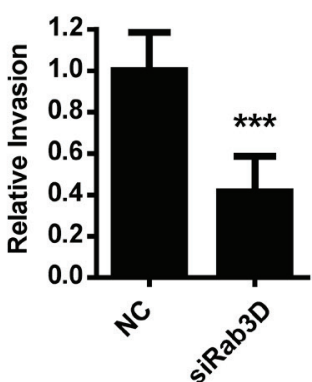

D
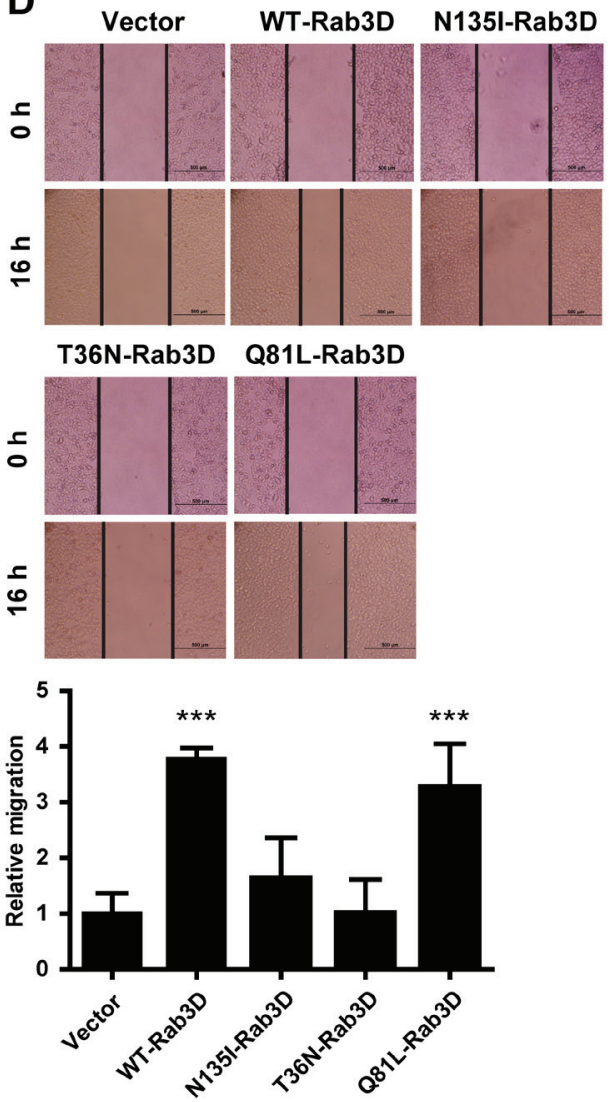

Figure 3: The effect of Rab3D on tumor cell migration and invasion in vitro. (A). Down-regulation of Rab3D markedly repressed tumor cells migration (wound healing assays). Representative images and quantification of migration assay in Rab3D knockdown MDA-MB-231 cells (Scale bar, $200 \mu \mathrm{m}$ ) or A375 cells (Scale bar, $50 \mu \mathrm{m}$ ). ${ }^{* *} p<0.01, * * * p<0.001$. (B). Down-regulation of Rab3D inhibited the invasion capability of tumor cells on Matrigel-coated transwell insets in the invasion assay. Representative images and quantification of invasion assay by MDA-MB-231 cells (Scale bar, $100 \mu \mathrm{m}$ ) or A375 cells (Scale bar, 50 $\mu \mathrm{m}$ ) in which Rab3D was depleted. $* * p<0.01, * * * p<0.001$. (C). The effect of Rab3D on MMP-2 and MMP-9 activity. Gelatin zymography analysis of MMP-2 and MMP-9 activity in CM from Rab3D knockdown MDA-MB-231 cells or Rab3D overexpressing MCF-7 cells. (D). Representative images and quantification of migration assay by MCF-7 cells transfected to express Rab3D mutation. Scale bar, $200 \mu \mathrm{m}$. $* * * p<0.001$. (E). Representative images and quantification of the invasive phenotype. Scale bar, $100 \mu \mathrm{m} . * * * p<0.001$. 
was observed when adding the recombinant Hsp90 $\alpha$ to the culture medium (Fig. 5I-5J and Fig.S3F-G). These results demonstrate that Rab3D promotes cancer cell migration and invasion via Hsp90 $\alpha$ release.

\section{Rab3D is necessary for tumor metastasis and progression in vivo}

To further test the significance of Rab3D in tumor progression, we next investigated the function of Rab3D in tumor metastasis and progression in vivo. At first, we established the cancer cell lines stably overexpressing recombinant human Rab3D (Fig. 6A and Fig. S4A) and stably transfecting with GFP-Rab3D shRNA, respectively (Fig. 6B and Fig. S4B). We injected these cells separately into mammary fat pads of nude mice to establish spontaneous metastasis model and monitored tumor growth and metastasis. Clear boarders without obvious invasion to surrounding tissues were observed in the control group, while tumors formed by Rab3D-MCF-7 cells invaded surrounding muscles, mammary fat pads as well as lung tissues (Fig. 6C and Fig. S4C), demonstrating that Rab3D-MCF-7 cells became more invasive. In the metastatic lung, we observed small metastatic clones with GFP density near the blood vessels in the Rab3DMCF-7 group. Conversely, stable knockdown of Rab3D in xenograft tumor model is sufficient to reduce metastatic colonies in the lung (Fig. 6D and Fig. S4D). In addition, there were no obvious differences of cell proliferation in vitro (Fig. $\mathrm{S} 5 \mathrm{~A}$ and $\mathrm{S} 5 \mathrm{~B}$ ) and tumor growth in vivo (Fig. S5C-F) among different groups, although the overexpression of Rab3D decreased apoptosis to some extent (Fig. S5G), demonstrating that Rab3D is highly specific for promoting tumor invasion and metastasis but not due to changes in proliferation.

Consistent with our observations in vitro, the levels of Rab3D expression were positively correlated with the levels of N-cadherin, phosphorylated GSK3 $\beta$, snail, but negatively correlated with E-cadherin in vivo by IHC analysis (Fig. 6E and Fig. S6A-E). These data have further emphasized the positive correlation between Rab3D and EMT. Fig. 6F also showed that the level of plasma Hsp90 $\alpha$ in Rab3D-MCF-7 xenografts was twice as much as that in the control group $(n=5)$. Furthermore, stable Rab3D knockdown in the xenografts showed much lower level of Hsp90 $\alpha$ in blood plasma compared with controls.

Taken together, our results support that Rab3D is necessary and sufficient to promote tumor metastasis. A working model for the effect of Rab3D on metastasis is shown in Fig. 6G.

\section{DISCUSSION}

We have demonstrated the critical role of Rab3D

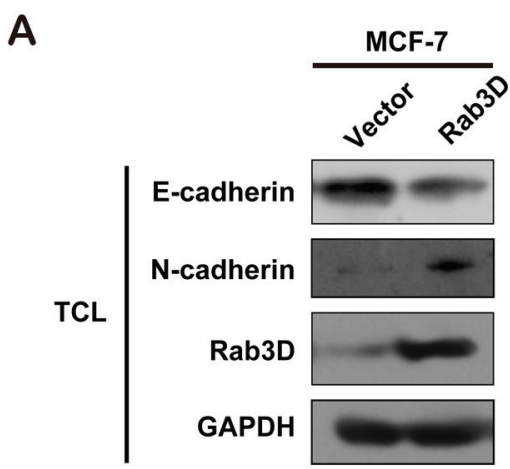

B

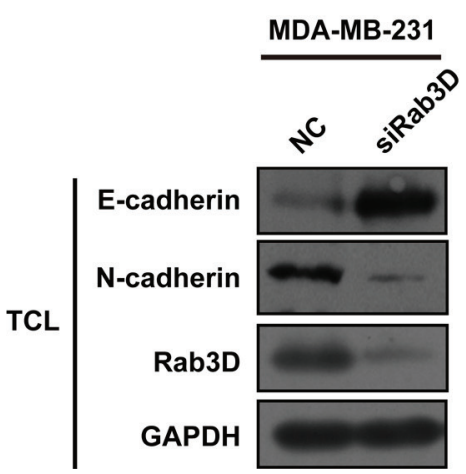

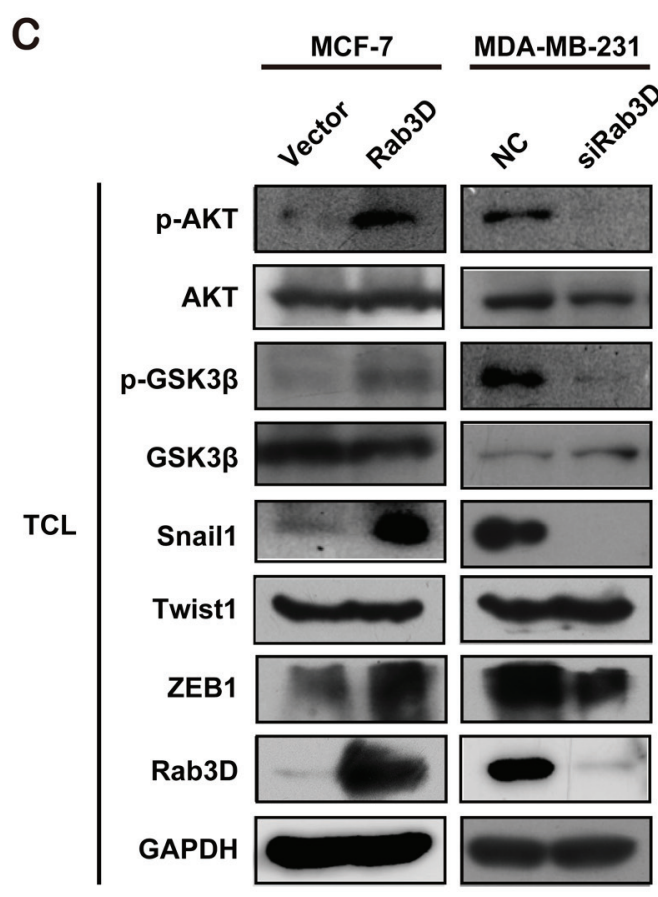

Figure 4: Rab3D as a regulator in EMT-like changes. (A). Western Blot analysis in Rab3D MCF-7 cells of EMT marker (E-cadherin and N-cadherin). (B). Measurement of E-cadherin or N-cadherin expression in siRab3D MDA-MB-231 cells. (C). The EMTrelated signaling activation (AKT/GSK3 $3 /$ Snail) in different group. 
A

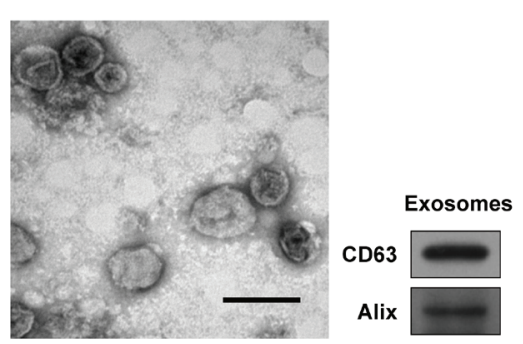

C
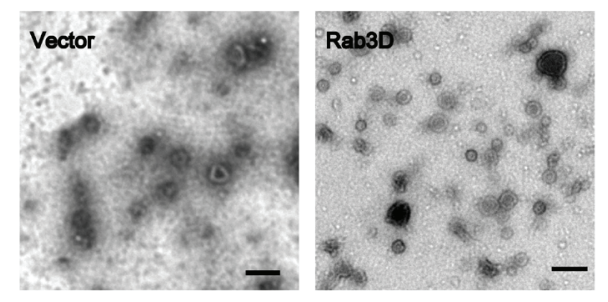

B

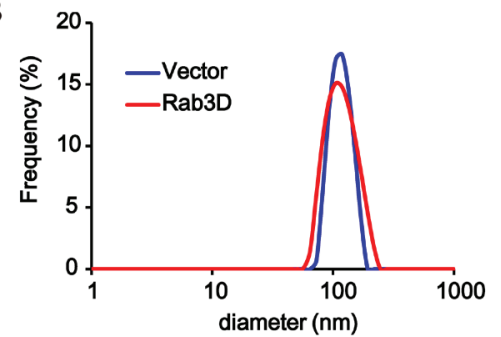

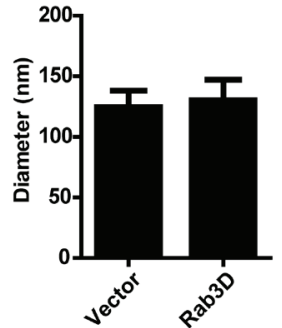

D

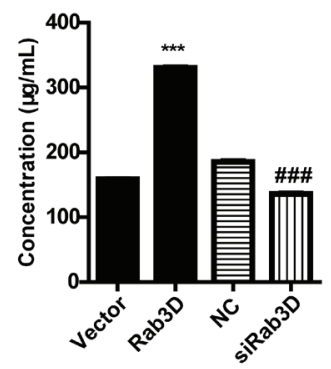

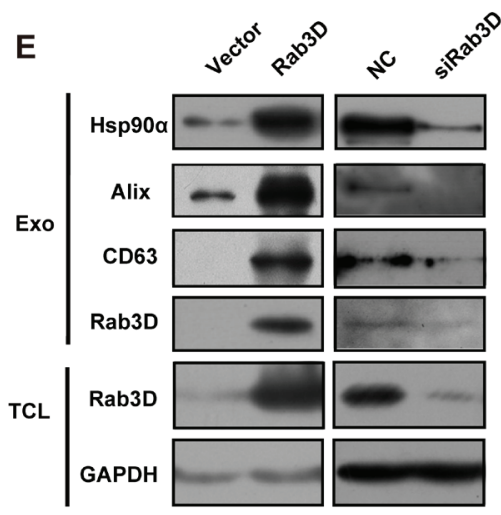

H

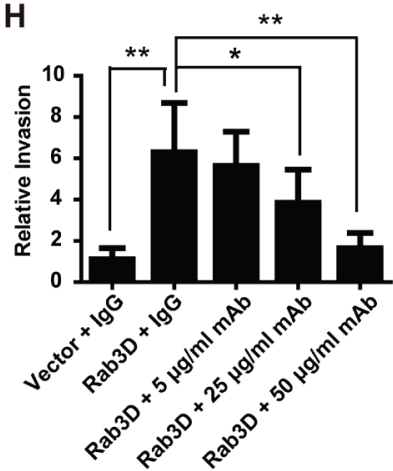

$\mathbf{F}$

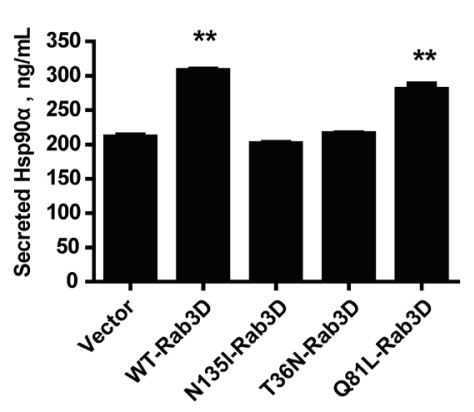

G

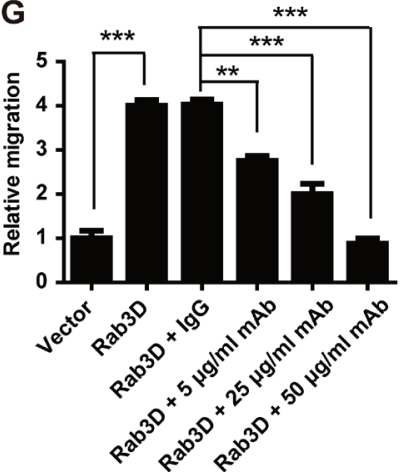

I
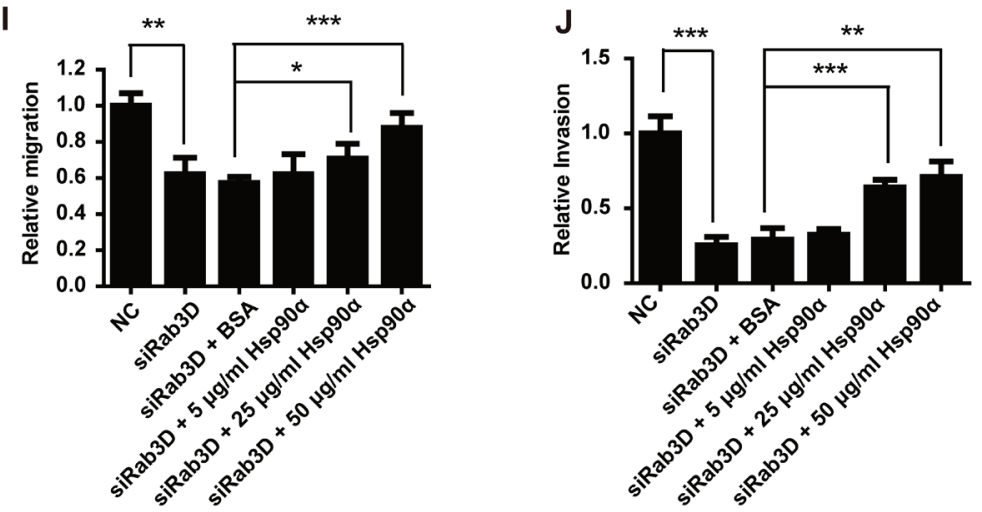

Figure 5: The role of secreted Hsp90a in Rab3D induced invasion. (A). Identification and characterization of exosomes. Exosomes were isolated by sequential centrifugations from supernatants. Scale bar, $100 \mathrm{~nm}$. An inset panel shows the exosomal marker, tetraspanin protein CD63 and Alix by Western Blot. (B). The size distribution of exosomes was analyzed by DLS and given as average \pm standard deviation $(\mathrm{n}=3)$. (C). Representative images and quantification of exosomes. Scale bar, $200 \mathrm{~nm}$. $* * * p<0.001$. (D). The total protein level of extracellular exosomes was detected by BCA $(n=3)$. (E). Western Blot analysis of exosomal marker CD63 and Alix in exosomes from Rab3D-MCF-7 cells or siRab3D-MDA-MB-231 cells. (F) The level of secreted Hsp90 $\alpha$ was detected by ELISA assay when the MCF-7 cells were transfected with different plasmid. (G). Quantification of migration assay of recombinant Hsp90 $\alpha$ treated MDA-MB-231 transfected with scramble RNA or Rab3D siRNA. Statistically significant $p$ values are indicated. ${ }^{* *} p<0.01, * * * p<0.001$. (H). Measurement of invasion in siRab3D MDA-MB-231 with or without addition of recombinant Hsp90 $\alpha{ }^{*} p<0.05, * * p<0.01,{ }^{*} * *$ $p<0.001$. (I). Quantification of migration assay. MCF-7 cells that expressed Rab3D, with or without Hsp90 $\alpha$ neutralizing antibody, were seeded. The relative migration distance was calculated after $16 \mathrm{~h} . * p<0.05, * * p<0.01, * * * p<0.001$. (J). Quantification of matrigel invasion assay. The effect of blocking extracellular Hsp90 $\alpha$ in MCF-7 cells over-expressing Rab3D on invasive ability. $* * p<0.01, * * * p$ $<0.001$. 

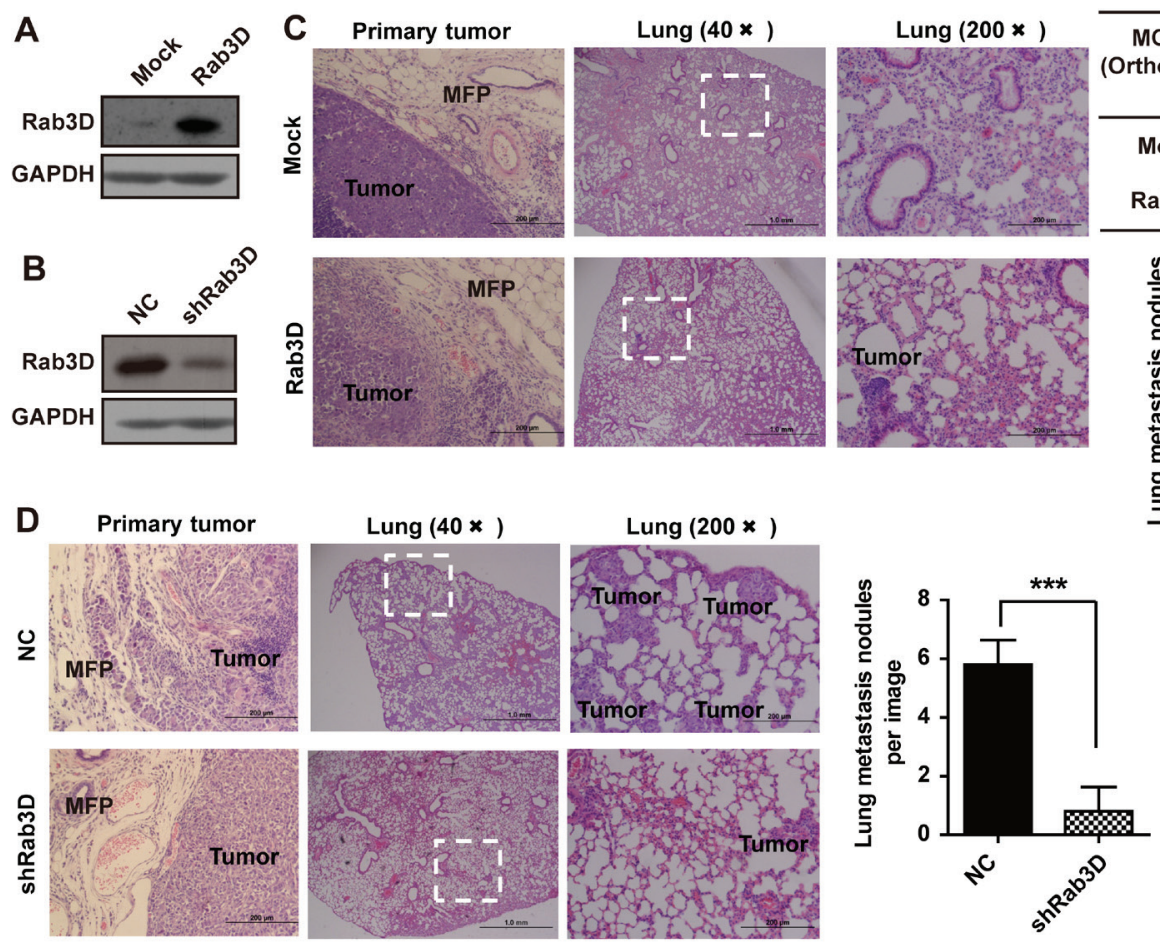

\begin{tabular}{ccc}
\hline $\begin{array}{c}\text { MCF-7 } \\
\text { (Orthotopic) }\end{array}$ & $\begin{array}{c}\text { Primary } \\
\text { tumor }\end{array}$ & $\begin{array}{c}\text { Metastasis } \\
\text { incidence } \\
\text { (Lung) }\end{array}$ \\
\hline Mock & $5 / 5$ & $0 / 5$ \\
Rab3D & $5 / 5$ & $5 / 5$ \\
\hline
\end{tabular}
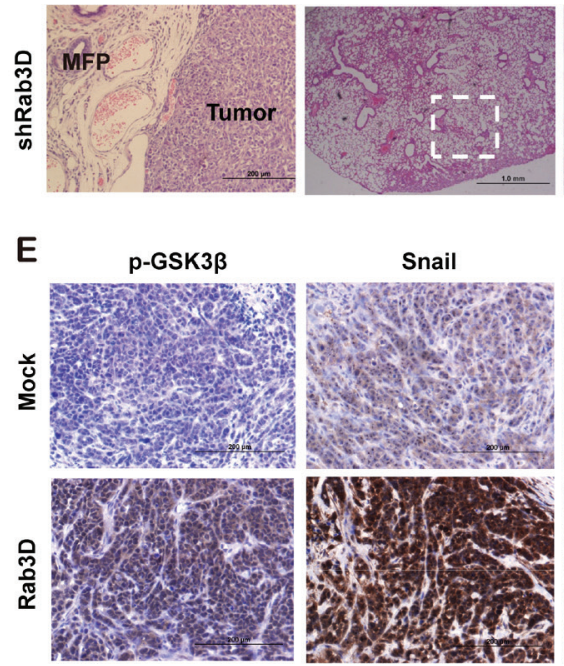

$\mathbf{F}$

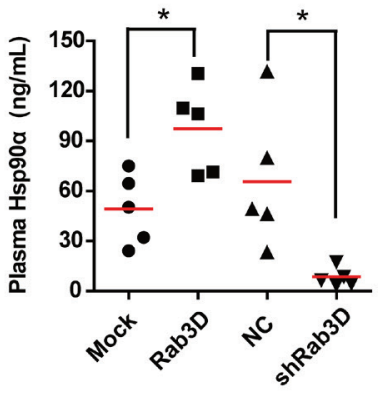

ZEB1

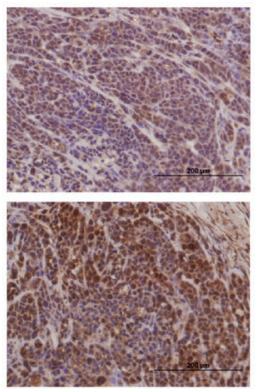

G
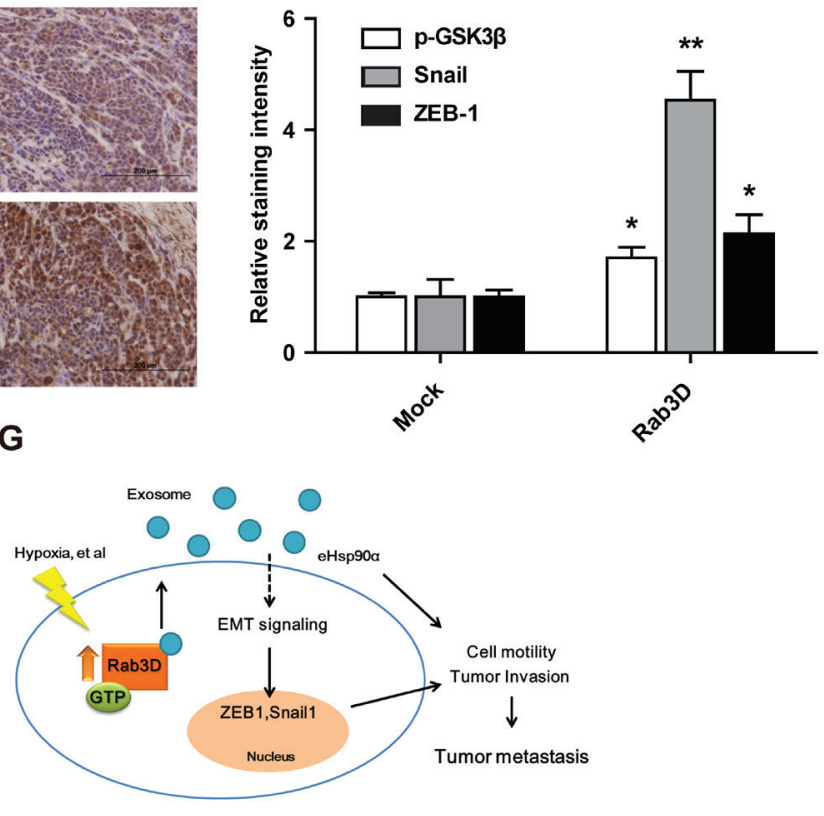

Figure 6: In vivo effects of Rab3D on tumor metastasis. (A-B). Rab3D expression in stably transfected tumor cell lines. (C). Representative images of primary tumors and lung section stained with hematoxilin and eosin in control and Rab3D-MCF-7 xenografts bearing mice ( $n=5$ mice per group). (D). H \& E staining of lung in control and shRab3D-MDA-MB-231 xenografts bearing mice. (E). Immunohistochemical staining images and quantification of EMT-related signaling activation in control and Rab3D-MCF-7 xenografts. Scale bar, $50 \mu \mathrm{m}$. (F). The level of plasma Hsp90 $\alpha$ in nude mice detected by ELISA assay ( $\mathrm{n}=5$ mice per group). (G). The working model for Rab3D-induced tumor cell invasion. In response to hypoxia, intracellular Rab3D is increased and its expression is correlated with tumor malignancy. Rab3D regulates exosomes release and Hsp90 $\alpha$ secretion, which promotes EMT and tumor metastasis. eHsp90 $\alpha$ indicates extracellular Hsp90 $\alpha$. 
in the regulation of tumor cell motility, invasion and metastasis. We have reported, for the first time, that the levels of Rab3D are elevated in clinical patient samples with many tumor types including breast, prostate, lung, colon, ovary, liver and skin cancers and are highly correlated with tumor TNM staging. Furthermore, the suppressions of Rab3D expression, which resulted in the deregulation of exosomes release and Hsp90 $\alpha$ secretion, have repressed tumor cell motility and invasiveness. These findings support the notion that Rab3D is a relevant diagnostic and therapeutic target against tumor progression.

\section{Rab3D can serve as a promising biomarker for tumor progression}

It has been reported that the patients with high Rab27A expression have a poor overall survival and is an independent prognostic marker for pancreatic ductal adenocarcinoma [37]. Moreover, the hepatocellular carcinoma patients with high level of Rab27A or Rab27B significantly reduce the overall survival [38]. In this study, the levels of Rab3D in the normal tissues or benign tumors are low, whereas the high levels of Rab3D are closely correlated with tumor TNM staging not grade, showing that Rab3D is a potential selective target for cancer progression. But we don't detect the correlation between Rab3D expression and the relative risk of death due to lack of clinical resources. Large scale, randomized, clinical trials are needed in the future to reveal that Rab3D is the valuable prognostic indicator for cancer patients in the clinic.

More importantly, the abnormal expression of Rab3D in cancer cells is not tissue- and tumor typespecific. The levels of Rab3D in breast, prostate, lung, colon, ovary, liver and skin carcinomas tissues are much higher than that in normal tissues with the IHC analysis, which suggests that Rab3D can be a broad-spectrum diagnostic biomarker for tumor progression in pathological examinations and refers the patients for diagnosis and treatment. Although 8 malignant cases had a Rab3D score of 0 , there were 92 cases that were positive and all of the normal tissues had the score of 0 , which indicated the good sensitivity and specificity of Rab3D as a diagnostic biomarker to detect carcinoma. On the other hand, our finding that the up-regulation of Rab3D protein levels in the majority of cancers appears inconsistent with the published data from Hendrix A and his co-workers, who reported that there was no difference of Rab3D in mRNA level in clinical breast cancer specimens [17]. But the inconsistence between mRNA and protein level changes implies that in the tumor cells there might be some important effectors to mediate the post-transcriptional regulation of Rab3D and its stabilization.

\section{Rab3D controls the exosomes release and Hsp90a secretion}

Rab27A and Rab27B GTPases can regulate the release of exosomes, which is an important process for metastatic signaling transduction $[15,16]$. In this study, we have found Rab3D as a new member to regulate exosomes. Rab3D can affect the total amount of exosomes but not their sizes. The roles of Rab3D have been described in Weibel-Palade body exocytosis, amylase release in exocrine cells and secretory vesicle traffic in lacrimal acinar cells [27, 31, 39]. But here, the association between Rab3D and exosomes release in tumor is uncovered for the first time. And exosomes have its roles in cancer development and metastasis [40]. Thus it is possible that Rab3D can promote tumor metastasis due to the secreted proteins from exosomes. Recently, large numbers of proteins in exosomes have been identified including adhesion molecules (e.g., integrin), membrane trafficking molecules (e.g., Rab proteins), chaperones (e.g., Hsp70 and Hsp90), signal transduction proteins (e.g., protein kinases), and immunosuppressive proteins (e.g., TGF- $\beta$ ) [41].

In this study, we focus on the important chaperoneHsp90 $\alpha$, which has no signal peptide. Hsp90 $\alpha$ is secreted via exosomes and strictly regulated by intracellular signaling pathways [42-44]. Secreted Hsp90 $\alpha$ can stabilize and therefore activate the function of MMPs [25, 45, 46]. We have demonstrated that Rab3D is essential for Hsp90 $\alpha$ secretion. More interestingly, after knocking down other secretion-related Rabs including Rab3B, Rab11A/B, Rab27A/B, Rab35 and Rab37, separately, knockdown of Rab3D resulted in the lowest Hsp90 $\alpha$ expression, suggesting the significant role of Rab3D in Hsp90 $\alpha$ secretion. Therefore, Hsp90 $\alpha$ might be mainly sorted into Rab3D-exosomes and finally released out of cells. Posttranslational modifications of Hsp90 contributions its function of chaperone [47] and the secretion of Hsp90 $\alpha$ is regulated by its phosphorylation of Thr90 [24]. Therefore, whether post-translational modifications control the Hsp90 $\alpha$ trafficking into exosomes, which deserves further study.

\section{Rab3D mediates EMT process and can be a novel option for anti-tumor metastasis}

EMT is the key player during tumor metastasis, in which E-cadherin is down-regulated and N-cadherin is increased $[48,49]$. Overexpression of Rab25 contributes to metastasis of bladder cancer through the induction of EMT [50]. We thus detected the EMT marker in Rab3D knockdown or overexpression tumor cells, and accordingly our data showed that Rab3D regulates EMT induction of tumor cells via the activation of Akt/GSK-3 $\beta /$ Snail pathway. One of the major reasons for Rab3D-mediated 
EMT is that the level of exosomes is increased. In fact, typical EMT hallmark proteins in cells have been found in exosomes, regulating the tumor microenvironment to promote the metastatic progression [51]. Furthermore, secreted Hsp90 has been reported as a driver of EMT events [52], which is consistent with our finding that secreted Hsp90 is essential for Rab3D-induced cancer cell migration and invasion. In this study, we believe Rab3D has its potential as a therapeutic target in cancer and can be a novel option to treat tumor metastasis. However, we also need to concern the side effects from a therapeutic perspective because Rab3D has its physiological role in granulocytes and alveolar epithelial cells [53].

In summary, our study provides an important theoretical foundation for us to understand the role of Rab3D in tumor progression and metastasis, suggesting that Rab3D is a potential diagnostic biomarker and a novel therapeutic target for anti-tumor metastasis. A better understanding of this biological function can lead to novel therapeutic strategies. In the future, we can develop smallmolecule inhibitors of Rab3D via rational drug design for the treatment of cancer.

\section{MATERIALS AND METHODS}

\section{Cell culture}

The methods of cell culture were performed as previously described [24]. Breast cancer cells MCF-7, MDA-MB-231 and melanoma cells B16-F0, B16-F1, B16-F10 (ATCC) were maintained in DMEM supplemented with $10 \%$ fetal bovine serum, $100 \mathrm{U} / \mathrm{ml}$ penicillin, and $100 \mu \mathrm{g} / \mathrm{ml}$ streptomycin (Hyclone). Breast cancer cells SKBr-3 were cultured in RPMI 1640 medium supplemented with $10 \%$ fetal bovine serum, $100 \mathrm{U} / \mathrm{ml}$ penicillin, and $100 \mu \mathrm{g} / \mathrm{ml}$ streptomycin (Hyclone).

\section{Transfection}

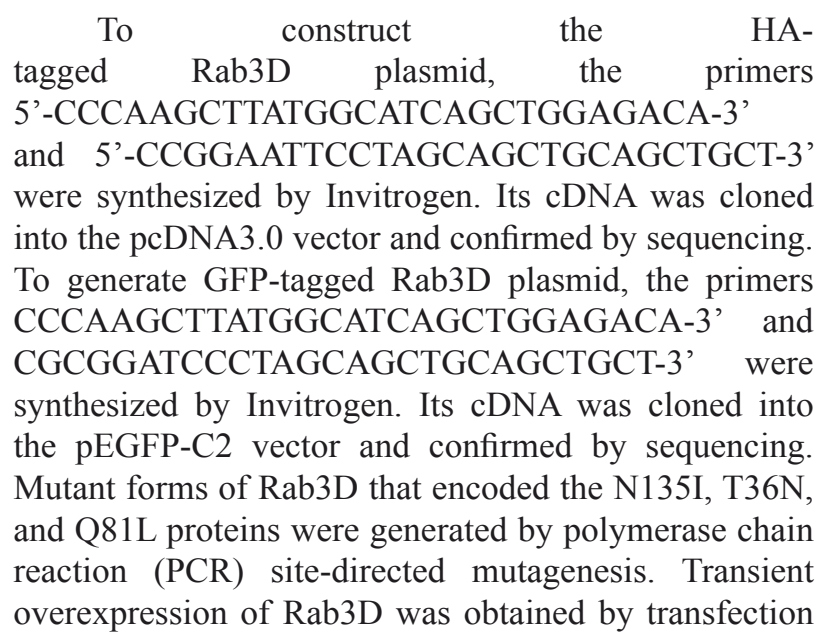

with HA-tagged Rab3D plasmid using the Turbofect agent (Thermo). Stable overexpression of Rab3D was obtained by Lentiviral transfection. Lentiviral Rab3D vector was purchased from Genepharm.

\section{RNA interference}

Down-regulation of Rab3D in MDA-MB-231 cells was obtained by transfection of its siRNA. The siRNAs for Rab3D were purchased from GenePharma. Scrambled siRNA was purchased from Gene-Pharma (Shanghai, China). The sequences of effective siRNA to repress Rab3D expression are listed as follows: Rab3D siRNA-1: 5'-GCAGCAGAUCAGAACUUCGACUAUA3'; Rab3D SiRNA-2: 5'-CGAACGUGUUGUGCCUGCUGAGGAU-3'.

The siRNAs were transfected with Lipofectamine 2000 reagent (Invitrogen) according to the manufacturer's protocol. Stable knockdown of Rab3D cells was generated by Lentiviral transfection of shRab3D (Genepharm). The sequence of effective shRNA for Rab3D is 5'-CGAACGUGUUGUGCCUGCUGAGGAU-3'. After $48 \mathrm{~h}$ transfection, Western Blot was applied to evaluate the knock-down efficiency.

\section{Western blot analysis}

Western Blot analyses were performed as previously described [24]. Cells were harvested, denatured, and subjected to SDS-PAGE. Proteins were transferred to polyvinylidene difluoride (PVDF) membrane, immunoblotted with the appropriated primary antibodies overnight at $4{ }^{\circ} \mathrm{C}$, incubated with horseradish peroxidase-conjugated secondary antibodies for $1 \mathrm{~h}$ at room temperature, then detected with an enhanced chemiluminescence system (Roche) according to the manufacturer's protocol.

\section{Immunofluorescence}

The cells were fixed in 4\% paraformaldehyde for $15 \mathrm{~min}$ at room temperature and blocked with $10 \%$ goat serum for $60 \mathrm{~min}$ at room temperature. Coverslips were then incubated at $4{ }^{\circ} \mathrm{C}$ overnight with the appropriate primary antibody (anti-Hsp $90 \alpha$ antibody). TRITC labeled phalloidin was used to visualize actin cytoskeleton. Images were photographed by Nikon microscope.

\section{Immunohistochemistry (IHC)}

Briefly, antigen retrieval was performed by heating for $15 \mathrm{~min}$ in $10 \mathrm{mmol} / \mathrm{L}$ sodium citrate buffer in a microwave oven. The paraffin-embedded tumor tissue slides were incubated with a primary antibody with an 
available dilution overnight at $4{ }^{\circ} \mathrm{C}$.

\section{Tissue microarray}

Breast cancer tissue microarray (BR1006), which contained 50 clinical female specimens (the age range is 19 - 82, including cancer adjacent normal breast tissue, fibro adenoma, cystosarcoma phyllodes, carcinosarcoma, medullary carcinoma, Paget's disease, mucinous adenocarcinoma and invasive ductal carcinoma), was purchased from Alenabio (Xi'an, China).

Cancer tissue microarray (MC246, Alenabio) contains 12 carcinomas (prostate, lung, colon, breast, ovary, uterine, esophagus, stomach, liver, kidney, skin and cerebrum) and their normal tissues near the cancer.

Breast cancer tissue microarray (BC081120) were purchased from Alenabio (Xi'an, China), that contained 110 clinical female specimens including cancer adjacent normal breast tissue and malignant tissues with different staging and grading.

Briefly, tissue sections were immunostained with anti-human Rab3D monoclonal antibody according to the protocol of immunofluorescent staining. The histochemical score is assessed by intensity of staining. For the intensity, the score index is 0 (negative), 1 (weak), 2 (moderate) and 3 (strong).

\section{Animal studies}

All animal studies were approved by the Institutional Animal Care and Use Committee of Tsinghua University. Tumor cells stably expressing or knocking down Rab3D mixed in Matrigel 1:1 were injected into the mammary fat pad of each mouse, respectively $\left(2 \times 10^{6}\right.$ cells per mouse $)$. Tumor volume was estimated by using the equation, $V=$ $0.5 \times a \times b^{2}, a$ is the length of the major axis of the tumor, and $b$ is the length of the minor axis.

For the metastasis analysis, lungs were extracted, weighed and fixed in 4\% paraformaldehyde for $4 \mathrm{~h}$, then paraffin embedded, sectioned, and applied to hematoxylin and eosin staining or IHC and Immunofluorescence assay for second tumor sections.

\section{Statistical methods}

Experimental results are expressed as mean value \pm SD. Statistically significant differences between groups were determined using a 2-tailed unpaired Student $\boldsymbol{t}$ test, where a $P<0.05$ is considered significant. The Chi-square test ( $\boldsymbol{x}^{2}$ test) was used to test for differences between categorical variables for the tissue microarray.

\section{ACKNOWLEDGMENTS}

We apologize that we can't cite all the related papers due to the limited space. We greatly thank the members of the Luo lab for insightful discussion and comments on the manuscript. We thank our member Feifei Qi for the guide of animal model. We also greatly thank Bipo Sun and Xuefei Yan for their contributions as the lab managers. This work was supported in part by the General Programs of the National Natural Science Foundation of China (No.81171998, 81272529, 81472667 and 81461148021), China's National Science and Technology Major Project for "Major New Drugs Innovation and Development" (2013ZX09509103).

\section{Authors' Contributions}

Conception and design: YZ Luo, J Yang

Development of methodology: J Yang, W Liu, XA

Lu, F Yan, L Li, YZ Luo

Acquisition of data: J Yang

Analysis and interpretation of data: J Yang

Writing, review, and/or revision of the manuscript:

J Yang, L Li, YZ Luo

Administrative, technical, or material support: J

Yang, W Liu, XA Lu, F Yan, L Li, YZ Luo

Study supervision: YZ Luo

\section{CONFLICTS OF INTEREST}

No potential conflicts of interest were disclosed.

\section{REFERENCES}

1. Valastyan S and Weinberg RA. Tumor metastasis: molecular insights and evolving paradigms. Cell. 2011; 147(2):275-292.

2. Chiang AC and Massague J. Molecular basis of metastasis. N Engl J Med. 2008; 359(26):2814-2823.

3. Hendrix A, Westbroek W, Bracke M and De Wever O. An ex(o)citing machinery for invasive tumor growth. Cancer Res. 2010; 70(23):9533-9537.

4. Vlassov AV, Magdaleno S, Setterquist R and Conrad R. Exosomes: current knowledge of their composition, biological functions, and diagnostic and therapeutic potentials. Biochim Biophys Acta. 2012; 1820(7):940-948.

5. D'Souza-Schorey C and Clancy JW. Tumor-derived microvesicles: shedding light on novel microenvironment modulators and prospective cancer biomarkers. Genes Dev. 2012; 26(12):1287-1299.

6. Valadi H, Ekstrom K, Bossios A, Sjostrand M, Lee JJ and Lotvall JO. Exosome-mediated transfer of mRNAs and microRNAs is a novel mechanism of genetic exchange between cells. Nat Cell Biol. 2007; 9(6):654-659. 
7. Hood JL, San RS and Wickline SA. Exosomes released by melanoma cells prepare sentinel lymph nodes for tumor metastasis. Cancer Res. 2011; 71(11):3792-3801.

8. Alderton GK. Metastasis. Exosomes drive premetastatic niche formation. Nat Rev Cancer. 2012; 12(7):447.

9. Thery C, Ostrowski M and Segura E. Membrane vesicles as conveyors of immune responses. Nat Rev Immunol. 2009; 9(8):581-593.

10. Stenmark H. Rab GTPases as coordinators of vesicle traffic. Nat Rev Mol Cell Biol. 2009; 10(8):513-525.

11. Goldenring JR. A central role for vesicle trafficking in epithelial neoplasia: intracellular highways to carcinogenesis. Nat Rev Cancer. 2013; 13(11):813-820.

12. Kim JK, Lee SY, Park CW, Park SH, Yin J, Kim J, Park JB, Lee JY, Kim H and Kim SC. Rab3a promotes brain tumor initiation and progression. Mol Biol Rep. 2014.

13. Tan PY, Chang CW, Chng KR, Wansa KD, Sung WK and Cheung E. Integration of regulatory networks by NKX3-1 promotes androgen-dependent prostate cancer survival. Mol Cell Biol. 2012; 32(2):399-414.

14. Wu CY, Tseng RC, Hsu HS, Wang YC and Hsu MT. Frequent down-regulation of hRAB37 in metastatic tumor by genetic and epigenetic mechanisms in lung cancer. Lung Cancer. 2009; 63(3):360-367.

15. Ostrowski M, Carmo NB, Krumeich S, Fanget I, Raposo G, Savina A, Moita CF, Schauer K, Hume AN, Freitas RP, Goud B, Benaroch P, Hacohen N, Fukuda M, Desnos C, Seabra MC, et al. Rab27a and Rab27b control different steps of the exosome secretion pathway. Nat Cell Biol. 2010; 12(1):19-30; sup pp 11-13.

16. Bobrie A, Krumeich S, Reyal F, Recchi C, Moita LF, Seabra MC, Ostrowski M and Thery C. Rab27a supports exosome-dependent and -independent mechanisms that modify the tumor microenvironment and can promote tumor progression. Cancer Res. 2012; 72(19):4920-4930.

17. Hendrix A, Maynard D, Pauwels P, Braems G, Denys H, Van den Broecke R, Lambert J, Van Belle S, Cocquyt V, Gespach C, Bracke M, Seabra MC, Gahl WA, De Wever $\mathrm{O}$ and Westbroek W. Effect of the secretory small GTPase Rab27B on breast cancer growth, invasion, and metastasis. J Natl Cancer Inst. 2010; 102(12):866-880.

18. Zhang JX, Huang XX, Cai MB, Tong ZT, Chen JW, Qian D, Liao YJ, Deng HX, Liao DZ, Huang MY, Zeng YX, Xie D and Mai SJ. Overexpression of the secretory small GTPase Rab27B in human breast cancer correlates closely with lymph node metastasis and predicts poor prognosis. J Transl Med. 2012; 10:242.

19. Hendrix A, Braems G, Bracke M, Seabra M, Gahl W, De Wever $\mathrm{O}$ and Westbroek W. The secretory small GTPase Rab27B as a marker for breast cancer progression. Oncotarget. 2010; 1(4):304-308.

20. Peinado H, Aleckovic M, Lavotshkin S, Matei I, CostaSilva B, Moreno-Bueno G, Hergueta-Redondo M, Williams C, Garcia-Santos G, Ghajar C, Nitadori-Hoshino A,
Hoffman C, Badal K, Garcia BA, Callahan MK, Yuan J, et al. Melanoma exosomes educate bone marrow progenitor cells toward a pro-metastatic phenotype through MET. Nat Med. 2012; 18(6):883-891.

21. Yang J, Song X, Chen Y, Lu XA, Fu Y and Luo Y. PLCgamma1-PKCgamma Signaling-Mediated Hsp90alpha Plasma Membrane Translocation Facilitates Tumor Metastasis. Traffic. 2014; 15(8):861-878.

22. Eustace BK and Jay DG. Extracellular roles for the molecular chaperone, hsp90. Cell Cycle. 2004; 3(9):10981100.

23. Picard D. Hsp90 invades the outside. Nat Cell Biol. 2004; 6(6):479-480.

24. Wang X, Song X, Zhuo W, Fu Y, Shi H, Liang Y, Tong $\mathrm{M}$, Chang $\mathrm{G}$ and Luo $\mathrm{Y}$. The regulatory mechanism of Hsp90alpha secretion and its function in tumor malignancy. Proc Natl Acad Sci U S A. 2009; 106(50):21288-21293.

25. Song X, Wang X, Zhuo W, Shi H, Feng D, Sun Y, Liang Y, Fu Y, Zhou D and Luo Y. The regulatory mechanism of extracellular Hsp90\{alpha\} on matrix metalloproteinase-2 processing and tumor angiogenesis. J Biol Chem. 2010; 285(51):40039-40049.

26. Schonn JS, van Weering JR, Mohrmann R, Schluter OM, Sudhof TC, de Wit H, Verhage M and Sorensen JB. Rab3 proteins involved in vesicle biogenesis and priming in embryonic mouse chromaffin cells. Traffic. 2010; 11(11):1415-1428.

27. Millar AL, Pavios NJ, Xu J and Zheng MH. Rab3D: a regulator of exocytosis in non-neuronal cells. Histol Histopathol. 2002; 17(3):929-936.

28. Bustos MA, Lucchesi O, Ruete MC, Mayorga LS and Tomes CN. Rab27 and Rab3 sequentially regulate human sperm dense-core granule exocytosis. Proc Natl Acad Sci U S A. 2012; 109(30):E2057-2066.

29. Qiu X, Valentijn JA and Jamieson JD. Carboxylmethylation of Rab3D in the rat pancreatic acinar tumor cell line AR42J. Biochem Biophys Res Commun. 2001; 285(3):708-714.

30. Wells A, Grahovac J, Wheeler S, Ma B and Lauffenburger D. Targeting tumor cell motility as a strategy against invasion and metastasis. Trends Pharmacol Sci. 2013; 34(5):283-289.

31. Chen X, Edwards JA, Logsdon CD, Ernst SA and Williams JA. Dominant negative Rab3D inhibits amylase release from mouse pancreatic acini. J Biol Chem. 2002; 277(20):18002-18009.

32. Kessenbrock K, Plaks V and Werb Z. Matrix metalloproteinases: regulators of the tumor microenvironment. Cell. 2010; 141(1):52-67.

33. Kalluri R and Weinberg RA. The basics of epithelialmesenchymal transition. J Clin Invest. 2009; 119(6):14201428.

34. Cochrane DR, Spoelstra NS, Howe EN, Nordeen SK and Richer JK. MicroRNA-200c mitigates invasiveness 
and restores sensitivity to microtubule-targeting chemotherapeutic agents. Mol Cancer Ther. 2009; 8(5):1055-1066.

35. Eustace BK, Sakurai T, Stewart JK, Yimlamai D, Unger C, Zehetmeier C, Lain B, Torella C, Henning SW, Beste G, Scroggins BT, Neckers L, Ilag LL and Jay DG. Functional proteomic screens reveal an essential extracellular role for hsp90 alpha in cancer cell invasiveness. Nat Cell Biol. 2004; 6(6):507-514.

36. Li W, Sahu D and Tsen F. Secreted heat shock protein-90 (Hsp90) in wound healing and cancer. Biochim Biophys Acta. 2012; 1823(3):730-741

37. Wang Q, Ni Q, Wang X, Zhu H, Wang Z and Huang J. High expression of RAB27A and TP53 in pancreatic cancer predicts poor survival. Med Oncol. 2015; 32(1):372.

38. Dong WW, Mou Q, Chen J, Cui JT, Li WM and Xiao WH. Differential expression of Rab27A/B correlates with clinical outcome in hepatocellular carcinoma. World J Gastroenterol. 2012; 18(15):1806-1813.

39. Zografou S, Basagiannis D, Papafotika A, Shirakawa R, Horiuchi H, Auerbach D, Fukuda M and Christoforidis S. A complete Rab screening reveals novel insights in WeibelPalade body exocytosis. J Cell Sci. 2012; 125(Pt 20):47804790.

40. Azmi AS, Bao B and Sarkar FH. Exosomes in cancer development, metastasis, and drug resistance: a comprehensive review. Cancer Metastasis Rev. 2013.

41. Yang $C$ and Robbins PD. The roles of tumor-derived exosomes in cancer pathogenesis. Clin Dev Immunol. 2011; 2011:842849.

42. McCready J, Sims JD, Chan D and Jay DG. Secretion of extracellular hsp90alpha via exosomes increases cancer cell motility: a role for plasminogen activation. BMC Cancer. 2010; 10:294.

43. Cheng CF, Fan J, Fedesco M, Guan S, Li Y, Bandyopadhyay B, Bright AM, Yerushalmi D, Liang M, Chen M, Han YP, Woodley DT and Li W. Transforming growth factor alpha (TGFalpha)-stimulated secretion of HSP90alpha: using the receptor LRP-1/CD91 to promote human skin cell migration against a TGFbeta-rich environment during wound healing. Mol Cell Biol. 2008; 28(10):3344-3358.

44. Rohl A, Rohrberg J and Buchner J. The chaperone Hsp90: changing partners for demanding clients. Trends Biochem Sci. 2013; 38(5):253-262.

45. Stellas D, El Hamidieh A and Patsavoudi E. Monoclonal antibody 4C5 prevents activation of MMP2 and MMP9 by disrupting their interaction with extracellular HSP90 and inhibits formation of metastatic breast cancer cell deposits. BMC Cell Biol. 2010; 11:51.

46. Yang Y, Rao R, Shen J, Tang Y, Fiskus W, Nechtman J, Atadja P and Bhalla K. Role of acetylation and extracellular location of heat shock protein 90alpha in tumor cell invasion. Cancer Res. 2008; 68(12):4833-4842.

47. Mollapour $\mathrm{M}$ and Neckers L. Post-translational modifications of Hsp90 and their contributions to chaperone regulation. Biochim Biophys Acta. 2012; 1823(3):648-655.

48. Lee JM, Dedhar S, Kalluri R and Thompson EW. The epithelial-mesenchymal transition: new insights in signaling, development, and disease. J Cell Biol. 2006; 172(7):973-981.

49. De Craene B and Berx G. Regulatory networks defining EMT during cancer initiation and progression. Nat Rev Cancer. 2013; 13(2):97-110.

50. Zhou BP, Deng J, Xia W, Xu J, Li YM, Gunduz M and Hung MC. Dual regulation of Snail by GSK-3beta-mediated phosphorylation in control of epithelial-mesenchymal transition. Nat Cell Biol. 2004; 6(10):931-940.

51. Tauro BJ, Mathias RA, Greening DW, Gopal SK, Ji H, Kapp EA, Coleman BM, Hill AF, Kusebauch U, Hallows JL, Shteynberg D, Moritz RL, Zhu HJ and Simpson RJ. Oncogenic H-ras reprograms Madin-Darby canine kidney (MDCK) cell-derived exosomal proteins following epithelial-mesenchymal transition. Mol Cell Proteomics. 2013; 12(8):2148-2159.

52. Hance MW, Dole K, Gopal U, Bohonowych JE, JezierskaDrutel A, Neumann CA, Liu H, Garraway IP and Isaacs JS. Secreted Hsp90 is a novel regulator of the epithelial to mesenchymal transition (EMT) in prostate cancer. J Biol Chem. 2012; 287(45):37732-37744.

53. van Weeren L, de Graaff AM, Jamieson JD, Batenburg JJ and Valentijn JA. Rab3D and actin reveal distinct lamellar body subpopulations in alveolar epithelial type II cells. Am J Respir Cell Mol Biol. 2004; 30(3):288-295. 